\title{
On the Development of Intention Understanding for Joint Action Tasks
}

\author{
Wolfram Erlhagen \\ Dept. Mathematics for $S \& T$ \\ University of Minho, Portugal \\ wolfram.erlhagen@mct.uminho.pt
}

\author{
Albert Mukovskiy \\ Russian Academy of Sciences \\ Moscow, Russian Federation \\ am@mct.uminho.pt
}

\author{
Fabian Chersi*† \\ *Dept. di Neuroscienze \\ Universita di Parma, Italy \\ fchersi@dei.uminho.pt
}

\author{
Estela Bicho \\ ${ }^{\dagger}$ Dept. Industrial Electronics \\ University of Minho, Portugal \\ estela.bicho@dei.uminho.pt
}

\begin{abstract}
Our everyday, common sense ability to discern the intentions of others' from their motions is fundamental for a successful cooperation in joint action tasks. In this paper we address in a modeling study the question of how the ability to understand complex goal-directed action sequences may develop during learning and practice. The model architecture reflects recent neurophysiological findings that suggest the existence of chains of mirror neurons associated with specific goals. These chains may be activated by external events to simulate the consequences of observed actions. Using the mathematical framework of dynamical neural fields to model the dynamics of different neural populations representing goals, action means and contextual cues, we show that such chains may develop based on a local, Hebbian learning rule. We validate the functionality of the learned model in a joint action task in which an observer robot infers the intention of a partner to chose a complementary action sequence.
\end{abstract}

Index Terms-intention understanding, social development, dynamic field model, mirror neurons, joint action in autonomous robots

\section{INTRODUCTION}

Many of our social activities during daily life rely on our ability to predict the consequences of other's behavior. We continuously interpret actions of our partners in terms of their intentions to adequately adjust our own actions. In adults, this form of social cognition includes the attribution of higher mental states like desires and beliefs. For younger children it is commonly believed that the goal inference process is restricted to the motor domain. Already preverbal children show the remarkable capacity of attributing goals to complex object-directed sequences like reaching-grasping-placing. As shown in imitation studies with adults as model, preverbal children are able to act on a goal that they had to infer since the end state of the sequence was hidden from view or the model 'accidentally' failed to place the object at a desired location (e.g., [1], [2]). In this paper, we focus on this lower level of understanding motor intention which, however, may be seen as a precursor for a higher mind reading capacity [3].

Many of the contemporary theories of action understanding rely on the notion that the observer uses his or her own motor repertoire to replicate the observed action and its effect. The discovery of the mirror neurons in premotor cortex (area F5) and parietal cortex ( $\mathrm{PF} / \mathrm{PFG}$ complex) of macaque provided the first neurophyisological evidence for the existence of such a matching system. Mirror neurons fire both when the monkey performs a goal-directed action such as grasping an object and when it observes another individual performing a similar action [3]. Using two learned reaching-graspingplacing sequences with specific goals (eating or placing), Fogassi and colleagues ( [4], [5]) have recently described activity patterns of mirror neurons in PF/PFG consistent with a model that postulates a chain between neurons coding subsequent motor acts. Crucial for an intention reading capacity, individual neurons encode identical motor acts dependent upon the ultimate goal of the sequence in which the act is embedded. Depending on the specific chain of mirror neurons that becomes activated by external events (e.g., an observed motor act) and contextual cues (e.g., object properties), the observer may thus predict what, most likely, the observed agent is going to do [5].

In this paper we address in a modeling study the fundamental question how the neural representations necessary for understanding complex action sequences may autonomously develop. In particular, we focus on the learning of action chains which become associated with goal representations and contextual cues during practice. The study complements and extends our previous work in the context of imitation and observational learning in which we have primarily focused on establishing a goal-directed mapping from action observation onto action execution (mirror properties; [6], [7]). We use the framework of dynamic neural fields to represent the activity of neural populations encoding different motor acts and goals. The synaptic links between any two populations in the distributed network are established using a neuroplausible, Hebbian learning dynamics. This learning rule also ensures that populations encoding motor acts split during practice into specific goal-directed subpopulations. Development thus manifests as a change within a complex dynamical system [8] which includes the emergence of new task specific representations.

Although the neurophysiological findings have to a large 
extent influenced the structure of the dynamic model, we do not intend here to explain details of the neural data. Instead, we validate the functionality of the model in a joint action task using a robot simulation environment.

The paper is structured as follows: In Sections II and III we discuss the concepts, underlying assumptions and mathematical details of the field model. The modeling results are presented in Section IV. We finish the paper with a critical discussion of modeling assumptions and results (Section V).

\section{MODEL CONCEPTS}

Our research group builds autonomous robots which interact in the context of a joint construction task in a natural and intelligent way with another agent, human or robot [9]. Using the dynamic field model as part of the robot control architecture, our ultimate goal is to endow the robots with a human-like intention reading capacity as a basis for nonverbal communication skills. To illustrate the 'developmental program' during the learning phase and the functionality of the mature system after learning we have chosen here a simple object directed reaching-grasping-placing scenario. The observing robot R1 has to select a complementary action sequence in dependence of the inferred action goal of the other agent R2. For instance, partner R2 may grasp an object of type $\mathrm{O} 1$ to place it in front of $\mathrm{R} 1$ with the intention to hand it over or, alternatively, may grasp the same object with the intention to directly plug it in as part of the complex object to be assembled by the team (see Fig. 1). To distinguish
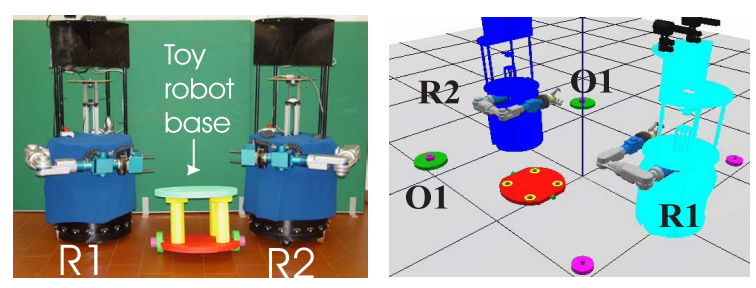

Fig. 1. On the left, two robots that have to cooperatively construct a toy robot base are shown. As a simple example to illustrate the functionality of the goal inference model, we have chosen the scenario illustrated on the right. Relevant are the objects labeled by $\mathrm{O} 1$. The observing robot $\mathrm{R} 1$ has to select a complementary object-directed action sequence in dependence of the inferred action goal of R2.

these two goals we call them in the following 'Grasping for Handing over (GfH)' and 'Grasping for Plugging (GfP)'. In the example, the selection of a particular chain is possible due to an additional contextual cue, the distribution of type $\mathrm{O} 1$ objects in the work-spaces of the two robots. If R2 is going to grasp $\mathrm{O} 1$ and no such object is within the reach of the partner, R2 will most likely start the handing over process.

Fig. 2 sketches the cognitive architecture for the observing robot R1 which is inspired by our current knowledge about the neuro-cognitive principles underlying the reasoning and decision processes in humans and other primates. A detailed discussion of the different layers with respect to the mirror circuit and prefrontal areas may be found in [6]. The central idea is that neuronal populations in the action observation layer (AOL) and the action simulation layer (ASL) encode motor primitives defined as whole object oriented motor acts such as reaching or grasping. Motor primitives do not encode the fine details of movements. They thus provide a sufficiently abstract level of description that allows for a matching between action observation and action execution across differences in embodiment, environmental constraints or motor skills. The existence of tool-use mirror neurons [10] can be seen as a direct physiological evidence for the existence of such a matching system in monkeys. Tool-use neurons fire both when the monkey grasps an object and when it observes a grasping action performed by the experimenter with an unknown tool. Using the DNF framework, we have recently shown [6] how the association to a movement not strictly in the motor repertoire of the observer may evolve during learning.

The neural populations in the goal layer (GL) become associated with the respective chains in ASL during practice. They provide input to the action execution layer (AEL) which also contains goal-directed chains. The input from GL biases R1's decision about a complementary motor behavior. Finally, layer OML represents memorized information about the location of objects in the workspaces of the two agents.

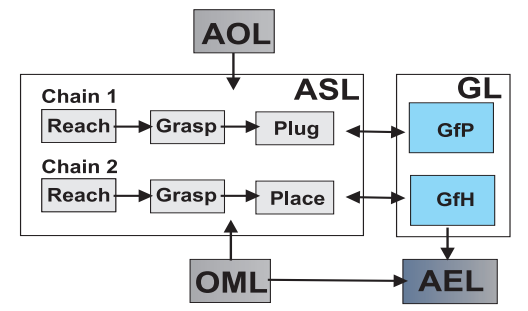

Fig. 2. Architecture for goal inference and selection of a complementary goal-directed motor response for the joint action task. The "Action Observation Layer" (AOL) encodes a visual description of the objet-directed motion of the partner (e.g., reaching, grasping, placing). The "Action simulation layer" (ASL) maps the perceived motor acts onto specific goaldirected action chains which have developed during learning and practice. The "Object Memory Layer" (OML) provides the contextual information about the objects in the workspace of each agent. The "Goal Layer" (GL) represents the inferred action goal of the partner. The "Action Execution Layer" (AEL) selects the appropriate goal-directed motor response based on 1) the inferred intention, and 2) the distribution of the objects in the workspace of the two robots. As part of a robot control architecture, this cognitive module gets input from the vision system. It is linked to the motor control system that provides the sequence of postures for the arm/hand/body to perform the selected goal-directed action (for details see [9]).

\section{Model Details}

\section{A. Dynamic neural field (DNF) model}

To model the dynamics of the different neural populations we use as a mathematical framework a discrete version of 
a dynamic neural field [11]. DNFs are ideally suited to represent two distinct but functionally related properties of neural populations in parieto-frontal areas: time integration of input signals and decision making [12]. For our motor sequence example this means, that for instance a grasping population as part of the GfH chain first integrates sensory and other evidence. This manifests in a continuous increase of population activity. When a certain activation threshold is reached, the population response becomes self-stabilized due to the increasing influence of excitatory interactions. At the same time, this representation engages in competition with the GfP grasping population due to lateral inhibition.

Each dynamic field represents a population of $2 N$ neurons which splits into an excitatory and an inhibitory subpopulation, each of dimension $N$. The activation of an excitatory and an inhibitory neuron $i$ at time $t, u_{i}(t)$ and $v_{i}(t)$, is governed by the following coupled system of differential equations (for model details see [13]):

$$
\begin{aligned}
\tau_{u} \frac{d}{d t} u_{i}(t) & =-u_{i}(t)+h+I_{i}(t) \\
& \left.+g\left(u_{i}(t)\right)\left[\sum_{j=1}^{N} w_{i j}^{u} f\left(u_{j}(t)\right)-v_{i}(t)\right)\right] \\
\tau_{v} \frac{d}{d t} v_{i}(t) & =-v_{i}(t)+g\left(v_{i}(t)\right)\left[\sum_{j=1}^{N} w_{i j}^{v} f\left(u_{j}(t)\right)\right]
\end{aligned}
$$

where the constants $\tau_{u}, \tau_{v}$ and $h<0$ define the time scales and the resting level of the dynamics, respectively. The firing rate $f($.$) and the shunting term g($.$) for the recurrent$ excitation are taken as non-linear functions of sigmoid shape. The interaction strength between any two neurons within the two subpopulation is defined by fixed synaptic weight functions, $w_{i j}^{u}$, and $w_{i j}^{v}$, which decrease as a function of the distance between the neurons. We have chosen Gaussian profiles with the specific choice $\sigma_{u}<\sigma_{v}$ and $A_{u}>A_{v}$ for the standard deviations and the amplitudes, respectively. This choice guarantees that the inhibition dominates over larger distances. The term $I_{i}(t)$ represents the summed input at time $t$ to the excitatory neuron $i$ (see below).

Within a certain range of field parameters, the excitatory population develops in response to an input above threshold strength, $A_{I}>A_{T H}$, the localized, transient activity pattern shown in Fig. 3. This transient behavior is the result of the recurrent excitation process which is counterbalanced by the local inhibitory feedback. It is important to stress that the inhibition may be tuned to guarantee an arbitrary slow decrease of the excitation. We use the extreme case of a selfstabilized, stationary peak to implement a working memory function (representations in OML and GL) [9].

\section{B. Hebbian learning}

We adopt a Hebbian perspective for the development of intention understanding [14] with a focus on establishing the
Panel A

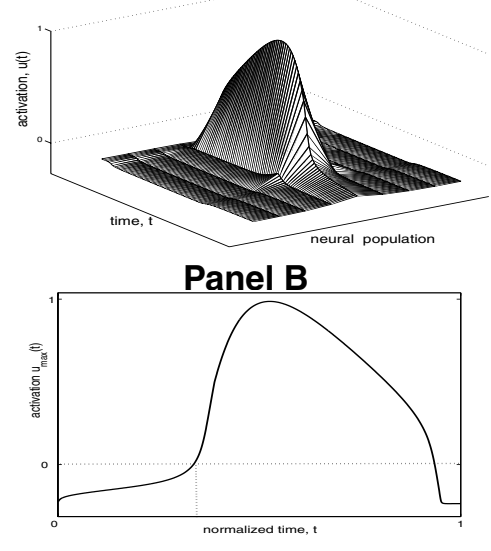

Fig. 3. Panel A: A localized transient activity pattern in a neural field consisting of $N=161$ neurons is shown. The external input defines the location of the pattern, the recurrent interaction parameters determine its width, that is, the number of neurons that become suprathreshold. Panel $B$ : The time course of the maximally activated neuron in the population is plotted. Time $t=0$ defines the onset of the external input. When the activation reaches the threshold $u=0$, the input integration period is over and the recurrent interaction processes start to dominate the population dynamics.

link between action means, contextual cues and goals. The learning process starts under the assumption that 1) all necessary motor primitives are already within the motor repertoire of the observer, and 2) that the congruent mappings of an action matching system have been established in previous experiments [6]. The Hebbian learning rule for increasing the synaptic efficacy, $s_{i j}$, between a 'presynaptic' neuron $j$ and 'postsynaptic' neuron $i$ belonging to different populations is given by (for a mathematical overview see [15]):

$\tau_{s} \frac{d}{d t} s_{i j}(t)=h\left(u_{i}(t)\right)\left[\alpha f\left(u_{j}(t)\right) f\left(u_{i}(t)\right)-s_{i j}(t)\right]-\beta s_{i j}(t)$

with a time scale $\tau_{s} \gg \tau_{u}$ much larger compared to the time scale of the field dynamics. All weights passively decay with a slow rate defined by $\beta>0$. In addition, the first term on the right-hand side of (2) describes the usual Hebbian learning with scaling parameter $\alpha$ balanced by a memory decay. Both are shunted by a sigmoidal function $h\left(u_{i}\right)$ with threshold $u_{h}>u_{f}$. For a sufficiently activated postsynaptic neuron $u_{i}$, the decay or growth of the weight to a presynaptic neuron $u_{j}$ appears to be proportional to the factor $h\left(u_{i}\right)$. The accelerated weight decay in case of a silent $u_{j}$ is the responsible mechanism for the development of task specific subpopulations.

Once the learning period is over and the learned connections are mature, neuron $i$ gets from a population $k$ which is activated above threshold the time varying input $I_{i}^{k}(t)=$ $\sum_{j=1}^{N} s_{i j} f\left(u_{j}(t)\right)$. The total input to $i$ is computed by summing over all connected populations, $I_{i}(t)=\sum_{k} \gamma_{k} I_{i}^{k}(t)$, 
where $\gamma_{k}$ are weighting parameters to control how much each partial input contributes to the activation of the unit. For the integration property of the neural populations it is important that the total input is scaled to the threshold $A_{T H}$. This is achieved in the present simulations for simplicity by choosing adequate weight parameters $\gamma_{k}$ (in principle, a weight normalization term for the leaning rule could be used [15]). As a result, during action observation the propagation of activity in a chain is possible only if at least two information sources (e.g., preceding motor act and contextual cue) provide coherent input.

\section{Results}

For establishing the chains, we adopt a learning by observation paradigm [16] in which a teacher demonstrates the two sequences, each composed of three motor primitives (Fig. 2). Through learned mappings mappings [6], the observed motor primitives represented in layer AOL activate step by step the corresponding neural populations in the action simulation layer (ASL). To allow for Hebbian learning, the neural population responses encoding subsequent primitives must overlap in time. The data from the monkey experiment shows that this is indeed the case. Neurons classified as reaching, grasping or placing units show suprathreshold activity that lasts several 100 milliseconds [4]. In the model network with its strong synaptic excitatory reverberations, such time courses can be reproduced by adjusting the time scales of the dynamics accordingly (in a $75-100 \mathrm{~ms}$ range). The time course of a chain composed of three primitives is illustrated in Fig. 4 by showing the temporal profile of the maximally activated neuron in each population. During action observation, the chains

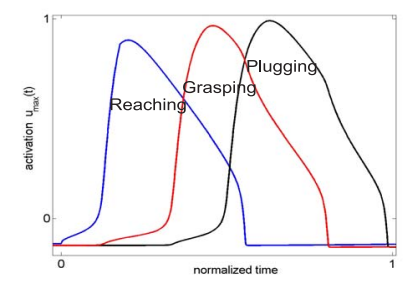

Fig. 4. The time courses of the transient neural population responses of the three motor primitives (reaching-grasping-plugging) forming a goal-directed chain are shown. During action observation, the chain can be triggered by partial visual information. It is maintained through the 'horizontal' synaptic connections and the integration of contextual cues.

may be triggered even if only partial visual information is available. Once the reaching population becomes active, for instance because the observer witnesses a hand approaching an object, the activity propagates to all synaptically coupled populations. However, only a population that appears to be already preshaped by additional information sources defining a particular chain will reach a suprathreshold activation level. Crucial for using the chain in a simulation mode for intention reading is the link to the goal or rewarded end state. In the field model, the two different goals of our construction example are represented by self-sustained activity bumps (working memory) of two competing populations in layer GL. Using the Hebbian rule, synaptic links are established between one of the goal representations (GfP or GfH), which is held in working memory for a specific learning block, and the transient motor representations in ASL. In Fig. 5 two snapshots of the learning process with alternating goal blocks are shown. In each snapshot, the top panel represents

snapshot $\mathbf{A}$

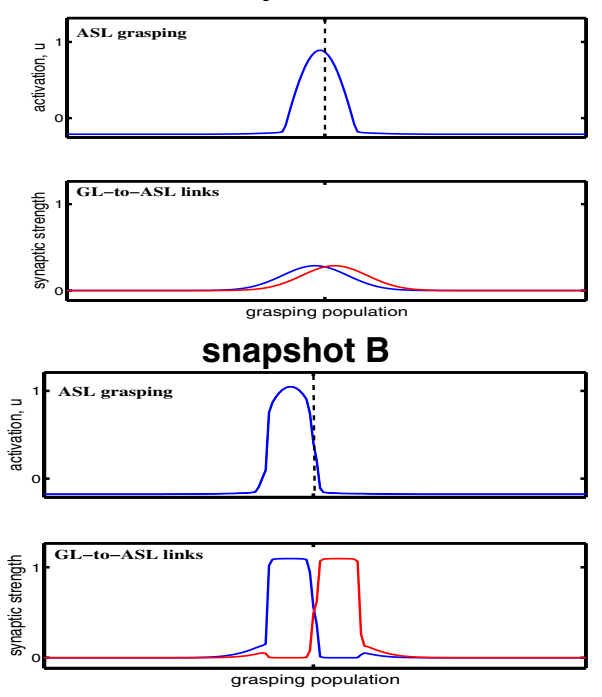

Fig. 5. The two snapshots illustrate the developing of goal-specific grasping neurons in the DNF model. Snapshot A represents the state before and Snapshot B the state after the learning period. On top, the maximal activity of the grasping population in a GfP trial is shown, on bottom the weight profiles from the maximally excited GfP (left) and GfH (right) neuron to the grasping population are plotted. Note that the influence of the initially weak input from the GfP population manifests in a slight shift of the population response to the left relative to the central line, the response in a $\mathrm{GfH}$ trial appears slightly shifted to the right (not shown). The interplay between the learning and the field dynamics continuously increases this shift during practice, leading to a nearly complete separation of the weight profiles and the population responses.

the activity of the grasping population. In the bottom panel, the two profiles represent the strength of the connections between the maximally excited neuron in the goal layer and the grasping population for the GfP population (left) and the $\mathrm{GfH}$ population (right). Initially, the synaptic connections are weak and the two distributions are centered over the population which is activated by external input (from ASL). However, due to a small spatial bias in the projections, the grasping population that becomes suprathreshold in a GfP trial does not coincide exactly with the active population in a GfH trial. The Hebbian learning rule leads to a continuous growth of the spatial bias since it penalizes the connections to grasping neurons which were initially stimulated by both goal representations. As a result of the learning, subpopulations 
of grasping neurons develop which are linked to a specific goal. Numerical tests with different initial conditions and block sizes show that the learning effect is robust as long as the initial weight distributions from GL do not completely overlap.

Associations between neural populations in ASL and populations representing contextual cues (in OML) are also established during practice using the Hebbian rule. This task knowledge is assumed to preshape the elements of the chain, expressing some expectation about possible action sequences. The impact on the intention reading capacity is illustrated in Fig. 6 and Fig. 7 in the context of the construction task. In both videos, R1 observes the hand of R2 approaching an object of type $\mathrm{O} 1$ (which is relevant according to the construction plan known to the agents). In this simple example, the contextual cue is the location of $\mathrm{O} 1$ objects in the workspace of the two robots. The location of each object is represented by a self-sustained activity bump in a neural field (OML layer, not shown) parameterized by the distance relative to $\mathrm{R} 1$. If all objects of type $\mathrm{O} 1$ are in the workspace of R2, the contextual cue supports the 'GfH' hypothesis. If at least one $\mathrm{O} 1$ object is in the workspace of $\mathrm{R} 1$, the 'GfP' hypothesis is supported. As shown by the activity pattern in the Goal Layer (GL), R1 interprets via the internal simulation mechanism the identical motor act displayed by $\mathrm{R} 2$ as belonging to different goal-directed sequences. The goal representation then biases through synaptic links the selection of complementary object-directed chains in AEL.

\section{Discussion AND OUTLOOK}

Intention understanding is considered a fundamental cognitive skill for all species living in social groups. It is currently an important topic of interdisciplinary investigation within the cognitive sciences and the domain of social robotics (for recent overviews see [17], [18]). We believe that adopting a developmental perspective may be especially enlightening by providing new insights about the plausibility of requirements and mechanisms for intention understanding discussed in the literature. The DNF model presented a neuro-cognitive inspired account of how the capacity to identify others' motor intentions from their motions might develop through a direct interaction with another agent. Our simulations showed that the neural representations implementing key mechanisms like motor simulation and cue integration may emerge as the result of real-time interactions of local populations without the need to refer to a central developmental program. There are, however, two important assumptions we have made in our model account. First, to develop a goal-directed chain during practice through Hebbian learning, the neural population in the goal layer (GL) must be activated above threshold. What external input might trigger this population? We suggest here that the initial motivation to get a reward is the driving factor

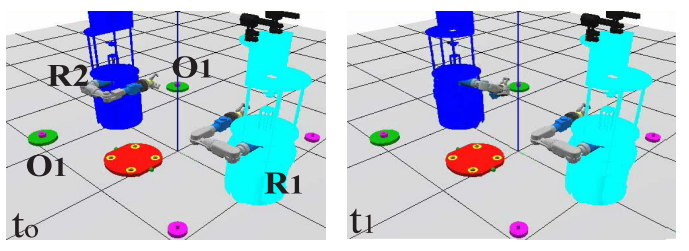

Goal (GL) Layer in R1

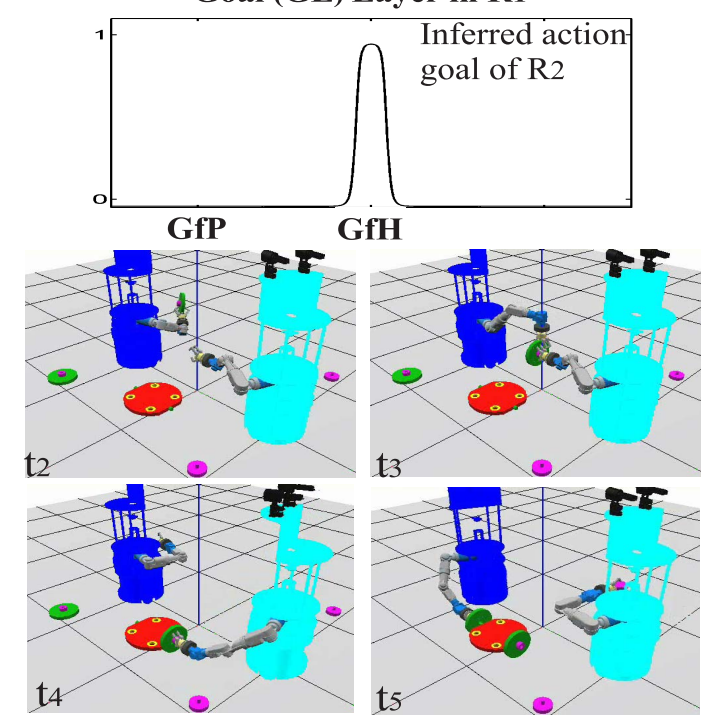

Fig. 6. Robot R1 observes partner R2 reaching for an object of type $\mathrm{O} 1$ $\left(t_{0}-t_{1}\right)$. All O1 objects are located in the workspace of partner R2. R1 thus infers that 'grasping the object for handing over (GfH)' is the current action goal of R2. This goal is represented in layer GL by a self-stabilized activity bump. As a result, R1 selects a complementary action sequence represented in layer AEL ('reaching towards the hand of R2-grasping-plugging', $t_{2}-t_{5}$ ).

for the learning agent (e.g., a monkey). A repeated execution of an action sequences in attempts to achieve that goal then strengthens the associations to the GL population. Second, it is assumed that the chains are established under the guidance of a teacher who shows the sequence. In the context of robotics and human development, a learning by observation approach is in general seen as an efficient means to reduce the dimensionality of the space in which a solution for a sensorimotor task has to be found [16]. One might speculate that the learning under the guidance of the teacher takes place in two steps. Initially, the focus is on establishing the links between chain elements to allow for a fluent execution of a particular action sequence like reaching-grasping-placing. Later during learning when it becomes clear that similar action sequences may have a different outcome (in terms of a reward), the focus shifts towards establishing the links to the goal and the contextual cues.

An important question that we have not addressed in this paper concerns how the abstract motor primitives (e.g., reaching, grasping) in layers ASL and AOL as a basis for action matching and goal inference may develop. Recent progress in the robotics domain suggest that agents may 

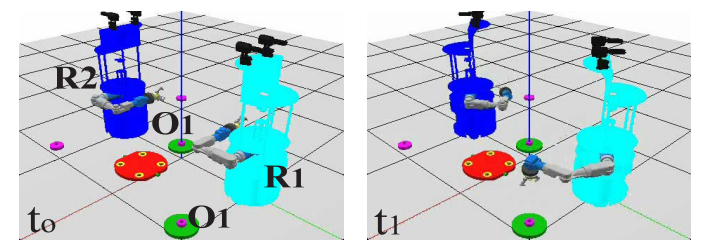

Goal (GL) Layer in R1

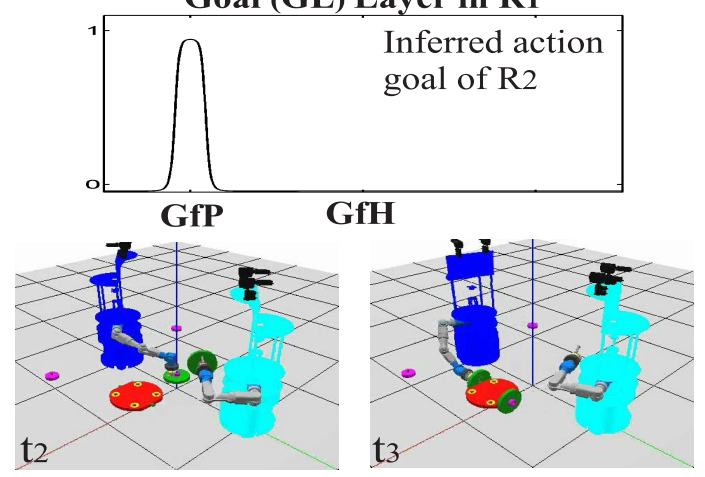

Fig. 7. Again, robot R1 observes R2 reaching for an object of type $\mathrm{O} 1\left(t_{0}\right.$ $\left.t_{1}\right)$. However, $\mathrm{R} 1$ has now also an $\mathrm{O} 1$ object in its workspace. R1 thus infers that the robot R1 most likely will grasp the object to directly plug it in (GfP) on its side of the construction. A 'GfP' action sequence directed towards object $\mathrm{O} 1$ in its own workspace represents the appropriate complementary behavior of R2 $\left(t_{1}-t_{3}\right)$.

autonomously learn and classify invariant sensorimotor behaviors by actively exploring the world though their bodies (e.g., [19], [20]). This approach is in particular appealing because it supports the idea of a co-development between sensorimotor and imitative capacities. In future work, we plan to explore in some detail the interplay between unsupervised and reinforcement learning techniques for developing the basic neural field representations.

To achieve the full complexity of a human-like intention reading capacity it is clear that the motor simulation circuit must depend on a host of different factors like sensory evidence and contextual information. The DNF model provides a Hebbian perspective on how such network may develop. Most importantly, the learning dynamics explains the existence of subpopulations that are linked to specific end states or goals.

As illustrated in the construction task example, the ASLGL-AEL circuit implements the idea that an observer may infer and adopt goals of others' in known task settings rather effortlessly. Once triggered by external inputs, the selforganizing properties of the circuit with its learned connections automatically lead to a selection of an adequate complementary behavior. Such an automatic processing clearly favors a fluent execution of joint action tasks. Currently, we are implementing and testing for the complex construction scenario with a relative large number of possible action sequences (Fig. 1) the capacity for automatic goal inference and action selection. The integration of additional information sources appears to be crucial for both subtasks.

\section{ACKNOWLEDGMENTS}

The present research was conducted in the context of the fp6-IST2 EU-project JAST-Joint Action Science and Technology (proj.nr. 003747). F. C. was supported in part by the grant MIUR prot. (proj.nr. 2004057380 004).

\section{REFERENCES}

[1] A. N. Meltzhoff and J. Decety, "What imitation tells us about social cognition: a rapprochement between developmental psychology and cognitive neuroscience," Phil. Trans. R. Soc. Lond. B, vol. 358, pp. 491-500, 2003

[2] H. Bekkering, A. Wohlschlger, and M. Gattin, "Imitation of gestures in children is goal-directed," The Quartely Journal of Experimental Psychology, vol. 53.

[3] V. Gallese, C. Keysers, and G. Rizzolatti, "A unifying view of the basis of social cognition," Trends in Cognitive Sciences, vol. 8, pp. 396-403, 2004.

[4] L. Fogassi, P. F. Ferrari, B. Gesierich, S. Rozzi, F. Chersi, and G. Rizzolatti, "Parietal lobe: from action organization to intention understanding," Science, vol. 308, pp. 662-667, 2005.

[5] F. Chersi, L. Fogassi, S. Rozzi, G. Rizzolatti, and P. F. Ferrari, "Neural chains for actions in the parietal lobe: a computational model," in Society for Neuroscience Abstracts, 2005, p. 412.8.

[6] W. Erlhagen, A. Mukovskiy, and E. Bicho, "A dynamic model for action understanding and goal-directed imitation." Brain Research, vol 1083, pp. 174-188, 2006.

[7] W. Erlhagen, A. Mukovskiy, E. Bicho, G. Panin, C. Kiss, A. Knoll, H. van Schie, and H. Bekkering, "Goal-directed imitation for robots: a bio-inspired approach to action understanding and skill learning," Robotics and Autonomous Systems, vol. 54, pp. 353-60.

[8] L. B. Smith and E. Thelen, "Development as a dynamical system." Trends in Cognitive Sciences, vol. 7, pp. 343-348, 2003.

[9] W. Erlhagen and E. Bicho, "The dynamic neural field approach to cognitive robotics," J. Neural Eng., vol. 3, pp. R36-R54, 2006.

[10] P. F. Ferrari, S. Rozzi, and L. Fogassi, "Mirror neurons responding to observation of actions made with tools in monkey ventral premotor cortex." Journal of Cognitive Neuroscience, vol. 17, pp. 1-15, 2005.

[11] S. Amari, "Dynamics of pattern formation in lateral-inhibitory type neural fields." Biological Cybernetics, vol. 27, pp. 77-87, 1977.

[12] X. J. Wang, "Probabilistic decision making by slow reverberation in cortical circuits," Neuron, vol. 36, pp. 955-968, 2002.

[13] D. Jancke, W. Erlhagen, H. R. Dinse, A. C. Akhavan, M. Giese, A. Steinhage, and G. Schöner, "Parametric population representation of retinal location: Neuronal interaction dynamics in cat primary visual cortex." Journal of Neuroscience, vol. 19, pp. 9016-9028, 1999.

[14] C. Keysers and D. I. Perrett, "Demystifying social cognition: a Hebbian perspective." Trends in Cognitive Sciences, vol. 8, pp. 501-507, 2004.

[15] W. Gerstner and W. Kistler, "Mathematical formulations of hebbian learning," Biol. Cybern, vol. 87, pp. 404-417, 2002.

[16] C. Breazeal and B. Scassellati, "Robots that imitate humans," Trends in Cognitive Sciences, vol. 6, pp. 481-487, 2002.

[17] D. A. Baldwin and J. A. Baird, "Discerning intentions in dynamic human action," Trends in Cognitive Sciences, vol. 5, pp. 171-178, 2001.

[18] B. Scassellatti, C. Crick, K. Gold, E. Kim, S. F, and G. Sun, "Social development," IEEE Computational Intelligence Magazine, vol. August 2006, pp. 41-47, 2006.

[19] Y. Demiris and A. Dearden, "From motor babbling to hierachical learning by imitation: a robot develomental pathway," in Proceedings of the Fith International Workshop on Epigenetic Robotics, L. Berthouze, Ed. Lund University Cognitive Studies 123, 2005, pp. 31-37.

[20] P. Andry, P. Gaussier, J. Nadel, and B. Hirshbrunner, "Learning invariant sensorimotor behaviors:A develomental appraoch to imitation mechanisms," Adaptive Behavior, vol. 12, pp. 117-140, 2004. 